\section{Énergie libérée par la machinerie de fusion SNAREpin}

David Tareste
Department of Physiology and Cellular Biophysics,

Columbia University,

1150 Saint-Nicholas avenue, New York 10032, États-Unis. dt2143@columbia.edu
> La fusion membranaire est un mécanisme ubiquitaire, qui est à la base de nombreux phénomènes physiologiques fondamentaux tels que la fécondation d'un ovule par un spermatozoïde, le développement musculaire, l'infection virale, ou encore l'ensemble du trafic vésiculaire au sein des cellules eucaryotes $[1,2]$. Tous ces processus impliquent que deux compartiments cellulaires, initialement séparés et chacun délimité par une membrane biologique, entrent en contact puis mettent en commun leurs composants lipidiques et cytosoliques. Les membranes biologiques étant des structures naturellement très stables, les événements de fusion ne peuvent se produire spontanément et nécessitent l'intervention de protéines spécialisées, qui vont aider à surmonter les barrières énergétiques de chaque étape, conduisant à la fusion membranaire [3]. Ces étapes comprennent: (1) le rapprochement des membranes; (2) la rupture de leur structure en bicouche (avec passage éventuel par un intermédiaire d'hémifusion où les feuillets externes des bicouches ont fusionné tandis que les feuillets internes demeurent séparés), et (3) la création d'un pore de fusion permettant le passage des composés solubles.

\section{Les SNARE orchestrent la fusion} membranaire lors du trafic vésiculaire Dans le cas du trafic vésiculaire intracellulaire, la fusion membranaire est orchestrée par les protéines SNARE (soluble $\mathrm{N}$-ethylmaleimide-sensitive factor attachment protein receptor), dont les plus étudiées et les mieux caractérisées sont les SNARE neuronales impliquées dans la fusion des vésicules de neurotransmetteurs avec la membrane plasmique présynaptique [4]. Un modèle mécanistique pour la fusion des vésicules synaptiques a émergé, et est désormais utilisé afin d'expliquer la fusion induite par les SNARE dans d'autres routes du transport intracellulaire [5]. Les vésicules sont tout d'abord arrimées à la membrane cible (target) par les protéines Rab et les facteurs d'attachement. Ensuite, un assemblage de type «fermeture éclair» entre la protéine $v$-SNARE, qui réside dans la membrane de la vésicule, et le com- plexe t-SNARE, situé dans la membrane cible, permet de rapprocher, déformer et ainsi mélanger les deux membranes. Ces complexes v-SNARE/t-SNARE, qui créent un pont transitoire entre les deux membranes destinées à fusionner et au sein desquels chaque SNARE est ancrée dans une bicouche distincte, sont appelés SNAREpins (ou complexes trans-SNARE). Lorsque la fusion est achevée, les complexes SNARE se retrouvent ancrés dans une seule et même bicouche, résultat de la fusion des deux, et forment une tresse d'hélices $\alpha$ complètement structurée (complexes cis-SNARE).

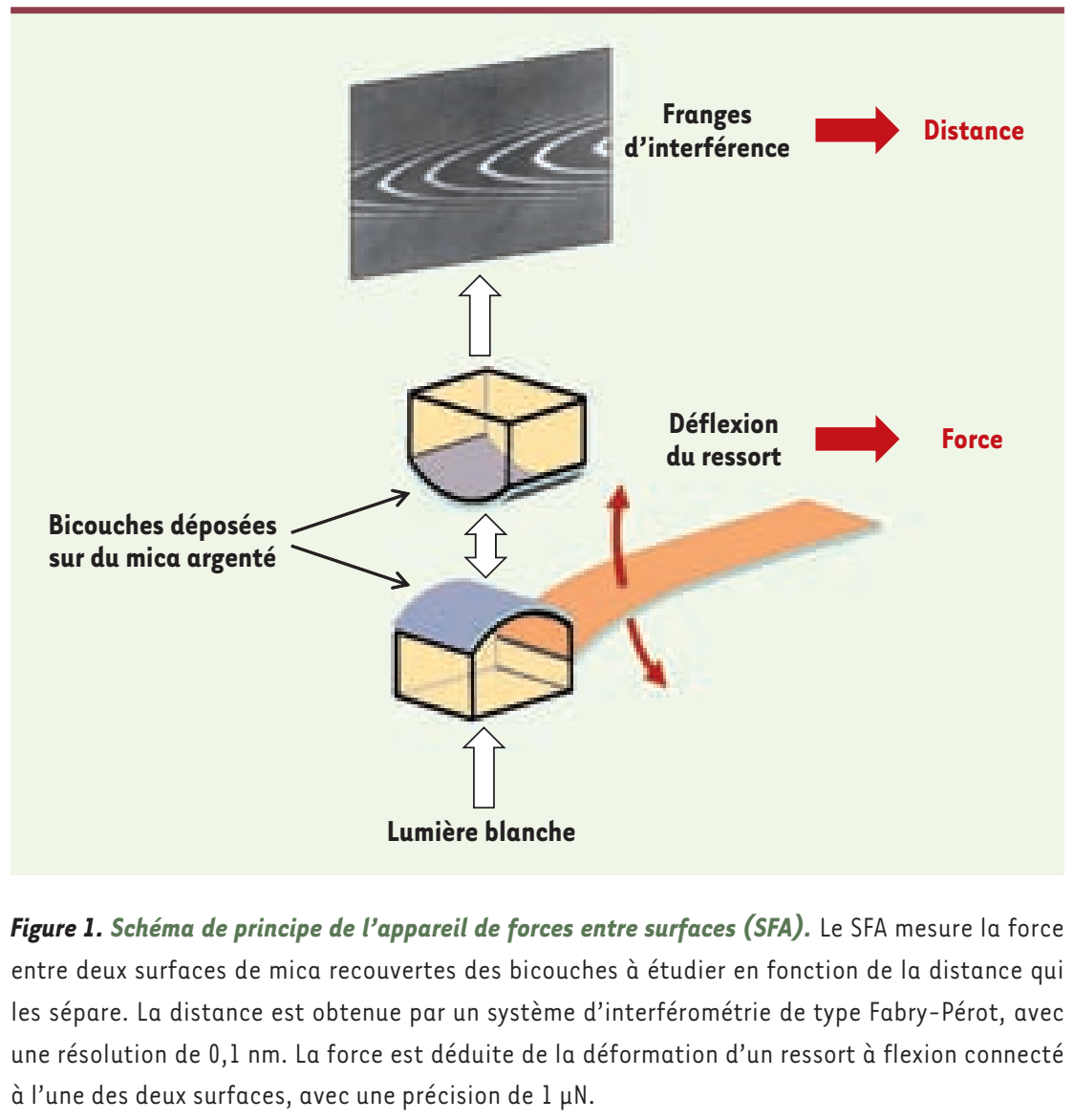


La plupart des études biochimiques et biophysiques réalisées à ce jour ont porté sur les complexes «post-fusion » cisSNARE [6]. Les complexes SNAREpins, en raison de leur caractère intrinsèquement dynamique, n'ont jamais pu être ni isolés, ni caractérisés. Quelle énergie est déployée durant l'assemblage des SNAREpins et comment cette énergie estelle transmise aux membranes afin de les rapprocher et les remodeler restent des questions actuellement sans réponse.

\section{Comment se forment} les complexes SNAREpins?

Pour répondre à cette question, il est nécessaire de pouvoir mesurer les interactions, à l'échelle nanométrique, entre SNARE incorporées dans des membranes lipidiques. Nous avons utilisé I'appareil de forces entre surfaces (SFA), qui permet de mesurer simultanément la distance et l'énergie d'interaction entre deux bicouches lipidiques fonctionnalisées [7]. Les surfaces d'étude du SFA sont deux feuillets de mica, placés dans une configuration de type cylindres croisés, dont une face est recouverte d'argent et l'autre de la bicouche lipidique. Un système de réflexions multiples de la lumière blanche sur les couches d'argent permet d'obtenir optiquement la distance, tandis que la déflexion d'un ressort plan relié à l'un des deux micas donne accès à la force entre les deux surfaces (Figure 1); cette force est proportionnelle à l'énergie d'interaction entre deux surfaces planes équivalentes. Au cours d'une expérience SFA, les surfaces sont tout d'abord rapprochées l'une de l'autre jusqu'au contact afin d'identifier la nature des interactions agissant à différentes distances, puis elles sont séparées afin de mesurer leur énergie d'adhésion.

Les profils d'interaction SFA entre vSNARE et t-SNARE insérées dans des bicouches lipidiques [8] ont révélé que les SNAREpins commencent à s'assembler Iorsque les membranes sont à environ $8 \mathrm{~nm}$ l'une de l'autre (Figure 2). In vivo, les protéines d'arrimage (Rab et facteurs d'attachement) devront donc positionner la vésicule à cette distance de la membrane plasmique cible afin que les SNARE puissent agir. L'énergie emmagasinée dans les SNAREpins - énergie qui pourra être utilisée pour la fusion - augmente au fur et à mesure que les membranes sont rapprochées l'une de l'autre et atteint son maximum lorsqu'elles se trouvent à $4 \mathrm{~nm}$ de distance. Dans cette configuration, les SNAREpins sont partiellement déstructurés au niveau de leur partie carboxy-terminale, et constituent un complexe très stable, irréversible à l'échelle de temps des phénomènes biologiques.

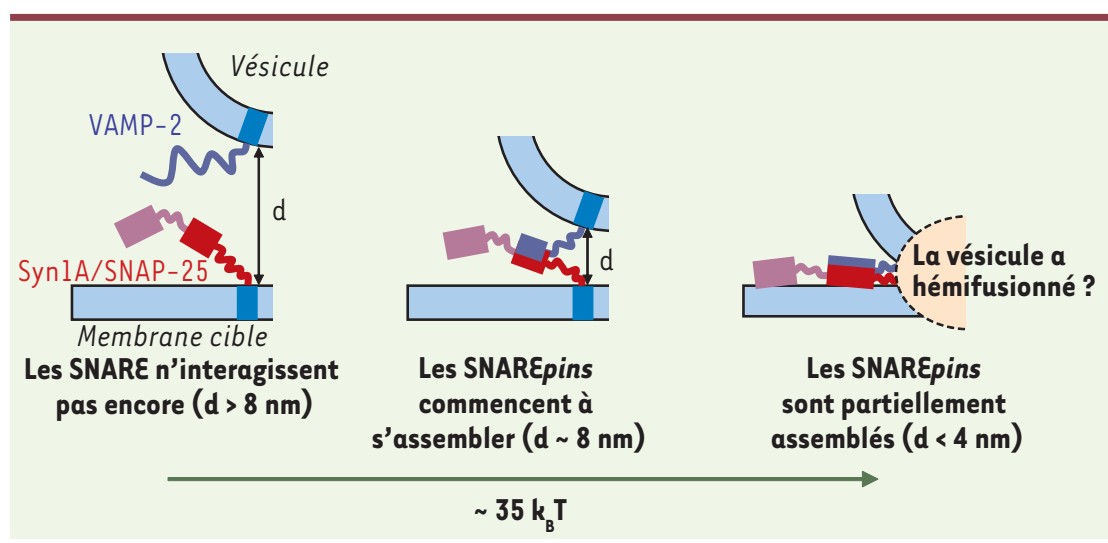

Figure 2. Dynamique et énergétique d'assemblage des SNAREpins. D'après nos mesures SFA, les protéines SNARE, initialement faiblement structurées, commencent à s'assembler sous la forme de complexes inter-membranaires SNAREpins lorsque les bicouches sont distantes de $8 \mathrm{~nm}$. L'énergie de ces SNAREpins augmente lorsque la distance inter-membranaire diminue, et culmine à $35 \mathrm{k}_{B} \mathrm{~T}$, une valeur très proche de l'énergie permettant de faire hémifusionner les membranes.
Ces complexes permettront donc de maintenir un contact très étroit entre les membranes durant l'organisation moléculaire de la fusion membranaire, et seront très probablement les substrats de certaines protéines de régulation, telles que Synaptotagmine et Complexine [9]. Nos mesures SFA montrent que l'énergie de ces SNAREpins partiellement assemblés $\left(35 k_{B} T\right)$ est très proche de l'énergie nécessaire à la formation des intermédiaires d'hémifusion (40-50 $\left.\mathrm{k}_{B} \mathrm{~T}\right)$, et que leur partie carboxy-terminale interagit physiquement avec la structure membranaire. Cette interaction permettra de transmettre l'énergie libérée durant l'assemblage des SNAREpins à la bicouche lipidique, et ainsi de favoriser la formation des structures intermédiaires de type non bicouche conduisant à la fusion. L'approche développée ici afin d'élucider la dynamique et l'énergétique d'assemblage des SNAREpins devrait pouvoir apporter bientôt des éléments nouveaux concernant le mode d'action des protéines de régulation, notamment comment ces protéines modifient la structure et l'énergie des SNAREpins et des membranes en apposition. $\diamond$

Energy of the SNAREpin

machinery for membrane fusion

\section{RÉFÉRENCES}

1. Galli T, Martinez-Arca S, Paumet F. Mécanismes de la fusion membranaire. Med Sci (Paris) 2002 ; 18: 1113-9.

2. Paumet F. La fusion cellulaire. Med Sci (Paris) 2003; $19: 663-4$.

3. Cohen FS, Melikyan GB. The energetics of membrane fusion from binding, through hemifusion, pore formation, and pore enlargement. J Membr Biol 2004 ; 199: 1-14.

4. Seagar M. Le complexe SNARE au cœur de la fusion membranaire. Med Sci (Paris) 2001 ; 17 : 669-74.

5. Weber T, Zemelman BV, McNew JA, et al. SNAREpins: minimal machinery for membrane fusion. Cell 1998 ; $92: 759-72$.

6. Brunger AT. Structure and function of SNARE and SNARE-interacting proteins. Quart Rev Biophys 2005 ; 38: $1-47$.

7. Israelachvili JN. Intermolecular and surface forces. London : Academic Press, 1992.

8. Li F, Pincet F, Perez $\varepsilon$, et al. Energetics and dynamics of SNAREpin folding across lipid bilayers. Nat Struct Mol Biol 2007 ; $14: 890-6$.

9. Melia TJ. Putting the clamps on membrane fusion: how complexin sets the stage for calcium-mediated exocytosis. Febs Lett 2007 ; $581: 2131-9$. 